\title{
Evaluación del uso de los inhibidores de la bomba de protones en un servicio de medicina interna
}

\author{
E. Martín-Echevarría, A. Pereira Juliá, M. Torralba, G. Arriola Peredaํㅜ, P. Martín Dávila², J. Mateos \\ y M. Rodríguez Zapata \\ Servicios de Medicina Interna y ${ }^{\prime}$ Pediatría. Hospital Universitario de Guadalajara. ${ }^{2}$ Servicio de Infecciosas. Hospital \\ Ramón y Cajal. Madrid
}

\section{RESUMEN}

Introducción: los inhibidores de la bomba de protones (IBP) bloquean la enzima $\mathrm{H}^{+} / \mathrm{K}^{+}$ATPasa en las células parietales gástricas, logrando la inhibición de la secreción de ácido clorhídrico de forma basal como tras estimulación. Las indicaciones apropiadas para su uso son: enfermedad por reflujo gastroesofágico, hemorragia digestiva aguda, úlceras activas, gastritis o esofagitis erosiva, dispepsia, gastropatía por AINE, profilaxis de úlcera de estrés en pacientes de riesgo.

El objetivo del estudio fue la revisión de las indicaciones de los IBP en nuestro medio y la evaluación de su utilización en el Hospital Universitario de Guadalajara.

Material y métodos: se realizó un estudio de corte transversal analítico con selección aleatoria de los pacientes atendidos en el Servicio de Medicina Interna durante todo el año 2003. Se revisaron un total de 208 historias de 832 pacientes (un 25\%) a los que se administraron IBP. La edad media fue de 67 años (rango 16-92), el 46,2\% fueron mujeres y las enfermedades subyacentes más frecuentes fueron: HTA, EPOC y DM.

Resultados: un 34,6\% de los pacientes tomaban IBP antes del ingreso. De estos, el 68,1\% no tenía indicación. Durante el ingreso se prescribió de forma inadecuada el IBP al 73,07\% y al alta, se mantenía el IBP sin indicación correcta.

Conclusiones: los resultados están en concordancia con los anteriormente publicados, manteniéndose una alta frecuencia de uso incorrecto de los IBP y siendo recomendable una utilización más racional para evitar los efectos secundarios, las interacciones con otros fármacos y aportar una atención médica más eficiente.

Palabras clave: Inhibidores de la bomba de protones. Indicaciones. Guía. Sobreutilización.

\begin{abstract}
Introduction: proton pump inhibitors (PPIs) block the $\mathrm{H}+/ \mathrm{K}+$ ATPase enzyme in gastric wall cells, leading to an inhibition of both baseline and stimulated acid secretion. Appropriate indications include: Gastroesophageal reflux, acute upper gastrointestinal bleeding, erosive gastritis or esophagitis, dyspepsia, NSAID-related gastropathy, and stress ulcer prophylaxis in high risk patients. The aims of this study were to review the current indications of PPIs, and to evaluate their use in Guadalajara's University Hospital.

Material and methods: a transversal, analytic, randomized study was carried out during 2003 in our internal medicine department. A total of 208 medical records for 832 patients receiving PPIs were reviewed (25\%). Mean age was 67 years (range: 16-92), 46.2\% were females, and most frequent conditions were HBP, COPD, and DM.

Results: $34.6 \%$ of patients took PPIs before admission, their use being inappropriate in $68.1 \%$ of them. Among hospitalized patients 73.03\% used PPIs inappropriately, and most of them had no such indication at discharge.

Conclusions: results are in accordance with the literature, with a high frequency of incorrect PPI use; a more accurate use of PPIs is to be recommended to avoid side effects and drug interactions, and to provide a more efficient medical care.
\end{abstract}

Key words: Proton pump inhibitors. Indications. Guidelines. Overuse.

Martín-Echevarría E, Pereira Juliá A, Torralba M, Arriola Pereda G, Martín Dávila P, Mateos J, Rodríguez Zapata M. Evaluación del uso de los inhibidores de la bomba de protones en un servicio de medicina interna. Rev Esp Enferm Dig 2008; 100: 76-81.

Recibido: 21-09-07.

Aceptado: 18-12-07.

Correspondencia: Esteban Martín-Echevarría. Servicio de Medicina Interna. Hospital General Universitario de Guadalajara. C/ Donantes de sangre, s/n. 19002 Guadalajara. e-mail: estebanmartinechevarria@yahoo.es

\section{INTRODUCCIÓN}

Los inhibidores de la bomba de protones (IBP) son fármacos que poseen un grupo benzomidazólico con elevada afinidad y actividad inhibidora que bloquea la enzima 
$\mathrm{H}^{+} / \mathrm{K}^{+}$ATPasa en las células parietales. Al ser de carácter básico, se mantienen en concentración más alta y mantenida en medio ácido. De este modo mantienen la inhibición en condiciones basales y en respuesta a estímulos, incluso en situaciones en que los antagonistas de receptores $\mathrm{H}_{2}$ y muscarínicos no lo consiguen $(1,2)$. Esto hace que sean fármacos ampliamente utilizados tanto en el ámbito hospitalario como extrahospitalario.

Los IBP tienen efectos secundarios como síntomas gastrointestinales $(<3 \%)$ (náuseas, diarrea y malestar abdominal), dolor osteomuscular y menos frecuentemente $(<0,7 \%)$ erupción cutánea, liquenoide, neuropatía periférica, ginecomastia y anemia hemolítica. Además presentan interacciones, por compartir la vía metabólica del citocromo P450, con otros fármacos como warfarina, diazepam y fenitoína, por lo que adecuando su uso se evitarán posibles complicaciones con dichos tratamientos $(1,2)$.

Aunque existen unas indicaciones bien definidas para su uso (1-4), no siempre se tienen en cuenta a la hora de prescribirlos (Tabla I). Diversos estudios publicados evidencian su sobreutilización (3-7), como en la profilaxis de la úlcera de estrés en pacientes de bajo riesgo (3-7) o concomitantemente al uso de corticoides $(8,9)$.

El objetivo de nuestro estudio es analizar la indicación de los IBP en la utilización ambulatoria previa al ingreso, durante el ingreso en el servicio de medicina interna y al alta hospitalaria.

\section{Tabla I. Indicaciones de uso de IBP}

-Enfermedad por reflujo gastroesofágico

-Hemorragia digestiva alta

-Tratamiento de enfermedad ulcerosa activa

-Tratamiento erradicador del H. pylori

-Esofagitis o gastritis erosiva

-Síndrome de Zollinger Ellison

-Dispepsia no ulcerosa

- Gastropatía por antiinflamatorios no esteroideos

-Profilaxis de la úlcera de estrés en pacientes de alto riesgo

Adaptado de Nardino y cols. (3), Noguerado Asensio y cols. (7) y Ponce Romero y cols. (4).

\section{MATERIAL Y MÉTODOS}

Se realizó un estudio de corte transversal, analítico, con selección de la muestra mediante aleatorización global, utilizando el programa de aleatorización de Excel (Excel 2003) para seleccionar al azar uno de cada 4 sujetos que fueron tratados con IBP en nuestro servicio durante el periodo de estudio. Se incluyeron 208 pacientes de un total de 832 que fueron tratados con IBP, al menos en una ocasión, independientemente de la dosis. El listado global de pacientes fue proporcionado por el sistema informático del servicio de farmacia hospitalaria.

El cálculo de tamaño muestral se realizó adjudicando un error alfa de 0,05 , un error beta de 0,10 , con el objeti- vo de detectar diferencias de hasta un $15 \%$ añadiendo la corrección de Fleiss. Se asumieron unas pérdidas del $15 \%$. Las historias que no pudieron ser recuperadas o en las que existía pérdida sustancial de información clínica se desestimaron y se incorporaron a la muestra, mediante aleatorización, nuevos sujetos. El periodo de estudio fue desde el 1-01-03 hasta el 31-12-03 y el ámbito del mismo, el Servicio de Medicina Interna del Hospital Universitario de Guadalajara, que cuenta con 400 camas. Se revisó la historia clínica de cada paciente, desde su llegada al hospital hasta que fue dado de alta, recogiendo los datos según el protocolo elaborado para el estudio, que incluye variables demográficas (sexo, edad) y clínicas (patología y tratamientos previos, motivo de consulta, tratamiento con IBP, etc.) (Tabla II).

Tabla II. Características demográficas

\begin{tabular}{lc}
\hline Número de pacientes & 208 \\
Edad media & 67 (rango: 16-92) \\
Sexo & $112(53,8 \%)$ \\
Hombre & $96(46,2 \%)$ \\
Mujer & \\
Patología concomitante & $47,1 \%$ \\
HTA & $10 \%$ \\
EPOC & $7 \%$ \\
DM & $7 \%$ \\
IRC & $62,2 \%$ \\
Otras (CI, Ictus, cirugía, FA,...) & \\
Tratamiento previo & $23,6 \%(49)$ \\
AINE & $4,3 \%(9)$ \\
Corticoides & $57,2 \%(119)$ \\
Otros & $14,9 \%(31)$ \\
No tratamiento & \\
\hline
\end{tabular}

HTA: hipertensión arterial; EPOC: enfermedad pulmonar obstructiva crónica; DM: diabetes mellitus; IRC: insuficiencia renal crónica; Cl: cardiopatía isquémica; FA: fibrilación auricular.

La indicación de los IBP fue determinada tras la revisión de la historia clínica y clasificada como correcta e incorrecta. Las indicaciones consideradas de utilización correcta de los IBP son las específicadas en el artículo de Nardino y cols. (3) y Ponce Romero y cols. (4) que se recogen en la tabla I. Como indicaciones incorrectas (Tabla III), se consideraron aquellas no presentes en la tabla previa. Se han agrupado en dos bloques principales; antiagregación/anticoagulación y otras, de acuerdo con lo establecido por otros autores (3).

Tabla III. Utilización de IBP sin indicación

-Profilaxis de úlcera de estrés en pacientes de bajo riesgo

-Terapia corticoidea

-Antiagregación/anticoagulación

-Hemorragia digestiva baja

-Sin razones

-Otras

Adaptado de Nardino y cols. (3), Noguerado Asensio y cols. (7) y Ponce Romero y cols. (4) 
Las variables continuas se representan mediante la media, la desviación estándar (DE) y el rango. Las variables cualitativas se expresan en forma de frecuencias absolutas y relativas. Para comparar las variables categóricas se utilizó la prueba de la $\chi^{2}$. Para la comparación de medias de muestras independientes se utilizó la t de Student o el análisis de la variancia. Se han considerado significativos valores de $\mathrm{p}<0,05$. Los análisis se han realizado con el paquete de programas estadísticos SPSS 11.0.1.

\section{RESULTADOS}

En el año 2003 hubo 1.660 pacientes ingresados en el Servicio de Medicina Interna del Hospital de Guadalajara, de los cuales 832 estaban en tratamiento con IBP, lo que supone un $50,12 \%$ de los pacientes. Los 208 pacientes seleccionados tenían una edad media de 67 años \pm 19,31 (rango:16-92), siendo 96 mujeres (46,2\%) y 112 hombres $(53,8 \%)$. Entre los antecedentes médicos previos al ingreso destacaron: hipertensión $(47,1 \%)$, enfermedad pulmonar obstructiva crónica $(10 \%)$, diabetes mellitus $(7 \%)$, insuficiencia renal crónica (7\%) y otras en un $62,2 \%$ (Tabla II).

La utilización de IBP no se asoció con la edad en los enfermos antes del ingreso, durante el mismo o al alta $(\mathrm{p}=0,76$ al ingreso, $\mathrm{p}=0,256$ planta y $\mathrm{p}=0,781 \mathrm{al}$ alta). Tampoco se halló relación con el sexo de los pacientes $(\mathrm{p}=0,206)$.

Un 23,6\% (49/208) de los pacientes antes del ingreso recibían tratamiento con antiinflamatorios no esteroideos (AINE), un 4,3\% (9/208) corticoides y un 57,2\% (119/208) eran polimedicados. Un 14,9\% (31/208) no tomaban medicación antes del ingreso.

\section{Tratamiento previo al ingreso}

El 34,6\% (72/208) de los pacientes estaba en tratamiento con IBP. El 68,1\% (49/72) no tenía una indicación adecuada de tratamiento (Fig. 1). Las indicaciones correctas

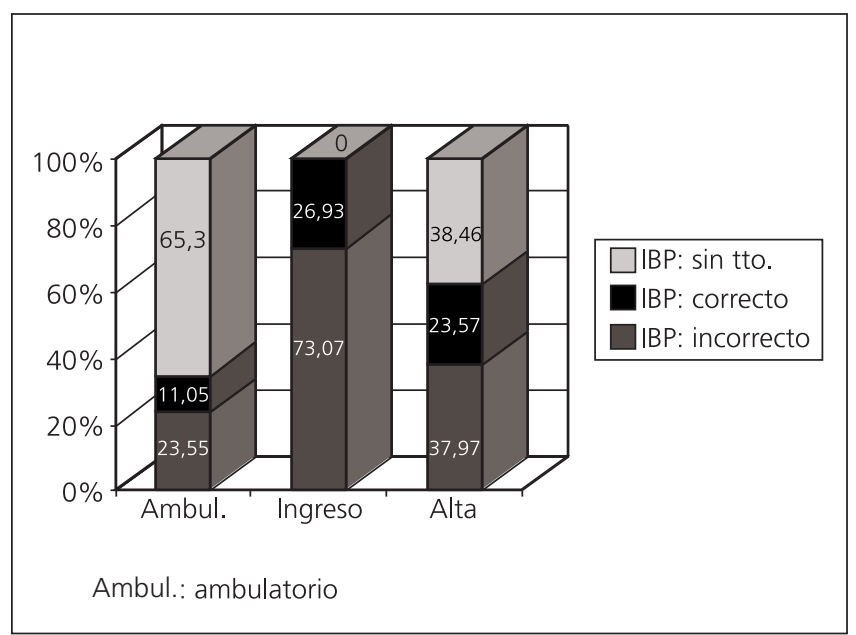

Fig. 1. Evolución del tratamiento con IBP en los pacientes.

más frecuentes fueron la gastropatía por AINE en un $11,1 \%(8 / 72)$, ulcus en el 9,7\% (7/72) y gastritis/enfermedad por reflujo gastroesofágico (ERGE) en el 8,3\% (6/72). Las causas incorrectas más frecuentes fueron el uso de antiagregación/anticoagulación en el 26,4\% (19/72) y otras razones en el 41,7\% (30/72). El 41,7\% (30) de los pacientes tomaba AINE; el uso correcto de IBP alcanzó el 43,3\% $(13 / 72)$, cuya indicación fue gastropatía por AINE $[11,1 \%$ (8) como tratamiento y $6,94 \%$ (5) como profilaxis].

El 6,9\% (5/72) tomaba corticoides sin indicación de protección gástrica en ninguno de los casos.

El 50\% (36/72) estaba polimedicado, sin indicación de uso de IBP en el 72,2\% de los casos (Tabla IV).

\section{Tratamiento en planta}

En la planta, los pacientes del estudio recibieron IBP, de los cuales el uso incorrecto alcanzó el 73,07\% (152/208) (Fig. 1). La indicación correcta más frecuente fue la profilaxis de la gastropatía por AINE en un 12,6\% (26/208), también por ulcus en el 7,7\% (16/208) y ERGE/gastritis en el 4,8\% (10/208). La razón más fre-

Tabla IV. Uso de los inhibidores de la bomba de protones

\begin{tabular}{|c|c|c|c|}
\hline & Antes del ingreso & En planta & Al alta \\
\hline $\begin{array}{l}\text { Utilización } \\
\text { Correcta } \\
\text { Razones } \\
\text { Incorrecta } \\
\text { Razones }\end{array}$ & $\begin{array}{c}72(34,6 \%) \\
23(31,9 \%) \\
\text { PG AINE } 8(11,1 \%) \\
\text { Ulcus } 7(9,7 \%) \\
\text { Gast/ERGE } 6(8,3 \%) \\
49(68,1 \%) \\
\text { AVA } 19(26,4 \%) \\
\text { Otras } 30(41,7 \%)\end{array}$ & $\begin{array}{c}208(100 \%) \\
56(26,93 \%) \\
\text { PG AINE } 26(12,6 \%) \\
\text { Ulcus } 16(7,7 \%) \\
\text { Gast/ERGE } 10(4,8 \%) \\
152(73,07 \%) \\
\text { AVA } 47(22,7 \%) \\
\text { Otras } 105(50,7 \%)\end{array}$ & $\begin{array}{c}128(61,5 \%) \\
49(38,3 \%) \\
\text { PG AINE } 24(18,7 \%) \\
\text { Ulcus } 16(12,5 \%) \\
\text { Gast/ERGE } 7(5,5 \% \%) \\
79(61,7 \%) \\
\text { A/A } 36(28,1 \%) \\
\text { Otras } 42(32,8 \%)\end{array}$ \\
\hline
\end{tabular}

PG AINE: profilaxis gastropatía por AINE; Gast: gastritis; ERGE: enfermedad por reflujo gastroesofágico; A/A: antiagregación/anticoagulación. 
cuente de uso incorrecto fue por antiagregación y anticoagulación en un $22,7 \%$ (47/208) y otras razones no establecidas en un 50,7\% (105/208) (Tabla IV).

\section{Tratamiento al alta}

Al alta, descendió su utilización hasta el $61,5 \%$ (128/208) de los pacientes, de los cuales, el $61,7 \%$ (79/128) no tenía indicación de uso (Fig. 1). Las indicaciones correctas más frecuentes también fueron la gastropatía por AINE en el 18,75\% (24/128), el ulcus en el $12,5 \%$ (16/128) y la gastritis/ERGE en el 5,5\% (7/128). Dentro de las causas no justificadas, la antiagregación y anticoagulación fue motivo de uso en el $28,1 \%$ (36) de los pacientes y el conjunto de otras razones fue del 32,8\% (42) (Tabla IV).

\section{Variación de tratamiento}

Se analizó la evolución del tratamiento con IBP desde que el paciente llegó al hospital hasta que era dado de alta; se mantuvo el tratamiento con IBP al 73,61\% de los pacientes, con una indicación inadecuada en el 62,27\%. Se introdujeron IBP al 58,6\% de ellos, con un uso inadecuado en el $61,34 \%$. Únicamente se retiró el tratamiento con IBP al $26,38 \%$ de los pacientes que no lo precisaban (Tabla V).

Tabla V. Variación en el tratamiento con IBP

\begin{tabular}{lccc}
\hline Ingreso & Planta & Alta & Adecuación alta \\
\hline IBP sí: & IBP sí: & IBP no: & \\
$72(34,6 \%)$ & $208(100 \%)$ & $19 / 72(26,38 \%)$ & \\
& IBP sí: & Inc: $33(62,27 \%)$ \\
& $53 / 72(73,61 \%)$ & Cor: $20(37,73 \%)$ \\
IBP no: & IBP sí: & Inc: $46(61,34 \%)$ \\
136 $(65,4 \%)$ & $75 / 136(58,6 \%)$ & Cor: $29(38,66 \%)$ \\
& IBP no: & \\
& & $61 / 136(41,4 \%)$ & \\
\hline
\end{tabular}

Inc: incorrecto; Cor: correcto.

El 54,83\% (17/31) de los $31(14,9 \%)$ pacientes que llegaron al hospital sin ningún tratamiento se fueron de alta con IBP. Su indicación fue correcta en el $41,17 \%$ $(7 / 17)$ por las siguientes razones: ulcus en el $17,64 \%$ (3/17), gastritis/ERGE en el 11,76\% (2/17) y gastropatía por AINE en el 11,76\% (2/17). En el 52,63\% (10/17) que no tenían indicación, los motivos fueron la antiagregación/anticoagulación en el 11,76\% (2/17) y otras razones en el 47,06\% (8/17).

La diferencia de los pacientes con IBP al alta frente al ingreso permite calcular que el riesgo de ser dado de alta con IBP tras un ingreso hospitalario tiene una odds ratio de 2,36 (IC95\%: 1,24-4,5) ( $\mathrm{p}=0,008)$. La indicación correcta de uso de IBP tras el alta hospitalaria es en un $6,4 \%$ $(\mathrm{p}=0,47)$ más adecuada que la obtenida previa al ingreso.

\section{Repercusión económica}

Los pacientes recibieron un total de 1.589 dosis de IBP, con una estancia media de 10,77 \pm 7,61 días, 514 intravenosas y 1.075 por vía oral, lo que supone un gasto de 7.573 euros, de los que el 73,07\% de los casos no estaba el uso indicado, lo que supone un gasto en exceso correspondiente a los 208 pacientes de 5.511,67 euros.

\section{DISCUSIÓN}

En este estudio se objetiva un uso incorrecto de los inhibidores de la bomba de protones, tanto en el ámbito intra- como extrahospitalario.

Nuestros resultados están en concordancia con los publicados en otras series (3-7). En la publicada por Nardino y cols. (3) y Noguerado Asensio y cols. (7), observamos, respectivamente, que el 54 y $72,2 \%$ de los pacientes recibieron fármacos supresores de la acidez gástrica (FSAG) durante el ingreso, de los que el 65 y $72,2 \%$ no tenían indicación. El 44 y 26,8\% recibían tratamiento previo al ingreso, y al alta lo continuaron el 35 y $65,9 \%$ de los pacientes, con un uso incorrecto en el 67 y $54,1 \%$ de los casos. En nuestro estudio, el 34,6\% de los pacientes tenía tratamiento con IBP siendo el $68,1 \%$ incorrecto. Durante el ingreso el 73,07\% tenía uso inadecuado y al alta se fueron con tratamiento IBP el $61,5 \%$ de los pacientes, con un porcentaje del $61,7 \%$ de uso incorrecto. Esto demuestra que la estancia hospitalaria no contribuye al uso correcto de los IBP o que la justificación de su utilización no viene bien reflejada en la historia clínica.

En nuestro estudio la profilaxis de la gastropatía por AINE fue la indicación correcta más frecuente en un $12,6 \%$ (10-15). Cualquier AINE aumenta el riesgo de sangrado, pero su efecto no es acumulativo y disminuye rápidamente con la suspensión del fármaco (12). Los IBP se han establecido como tratamiento preventivo a largo plazo y se ha demostrado que también son útiles en la profilaxis durante tratamientos cortos de AINE y aspirina, sobre todo en ancianos (16).

La causa más frecuente de utilización incorrecta no tiene una explicación clara aparente. En nuestro estudio, la más frecuente fue por la utilización de fármacos anticoagulantes y/o antiagregantes que conllevaba el uso de IBP como profilaxis del sangrado gastrointestinal. La indicación de IBP en este contexto no está clara; actualmente, Johnsen y cols. han publicado un estudio en el que los resultados indican que el uso de anticoagulantes orales se asocia a un incremento en el sangrado gastrointestinal, que aumenta más cuando se usa asociado a otros fármacos, aunque aún se debe precisar con más detalle cuáles son dichos fármacos y su modo de actuación (17). A su vez, el uso de antiagregación, incluso a dosis bajas, parece también relacionado con incremento en el riesgo de daño gastrointestinal $(16,18,19)$. 
El uso de corticoides $(8,9)$ está comúnmente asociado al uso de IBP como profilaxis de sangrado gastrointestinal, sin encontrarse evidencia científica que sugiera la necesidad de esta asociación. En los trabajos publicados por Conn y cols. (8) y Piper y cols. (9) no se encuentra asociación, aunque recientemente el grupo de Nielsen y cols. ha publicado la existencia de la relación entre corticoides, como único tratamiento, y el aumento de sangrado digestivo, con un riesgo relativo de 2,9 . El riesgo relativo disminuye a 1,9 en función del uso previo, de la edad, del sexo y de la patología subyacente (20), pero no recomiendan precauciones especiales salvo que existan antecedentes de sangrado. Existe evidencia de que la administración concomitante de corticoides con AINE sí produce un incremento del riesgo de hemorragia, por lo que en este caso la asociación con IBP está justificada, alcanzando un riesgo relativo de 9,8 en el estudio de Nielsen y cols. $(8,9,20)$.

Durante el ingreso hospitalario se encuentra un uso inadecuado del $73 \%$, dentro de las causas objetivables destacan, al igual que al ingreso y al alta, el uso de antiagregación y anticoagulación. En cuanto a razones no objetivadas, la profilaxis de la úlcera de estrés en pacientes de bajo riesgo es causa de instauración de IBP frecuente en los pacientes hospitalarios $(3,7)$, aunque este motivo no suele venir reflejado en las historias clínicas. Está demostrado que los pacientes críticos, con ventilación mecánica o alteración grave de la coagulación, tienen un riesgo incrementado de hemorragia gastrointestinal (2125), beneficiándose de la profilaxis de la úlcera de estrés. Este beneficio no se ha demostrado en otros casos, como en los pacientes postraumáticos en UCI (26) o de bajo riesgo (24). Otros estudios, sin embargo, hacen replantearse este hecho. Los resultados que obtienen sugieren que no existe un beneficio en la profilaxis de úlcera de estrés y sangrado gastrointestinal $(27,28)$. Incluso el uso de fármacos que aumentan el $\mathrm{pH}$ intragástrico podría incrementar el riesgo de neumonía nosocomial (28). Así, la profilaxis de rutina para el sangrado por estrés, incluso en pacientes de alto riesgo, parece no estar justificada y, de momento, ni la FDA ni la Agencia Europea para la Evaluación de Medicamentos han aprobado esta indicación profiláctica, aunque es un tema en debate $(27,28)$. En el periodo postoperatorio se ha objetivado una pequeña reducción significativa en el sangrado con la profilaxis farmacológica (29).

El ingreso de pacientes con pluripatología médica influye en que numerosos facultativos instauren de manera rutinaria tratamiento con IBP para profilaxis de bajo riesgo de daño en la mucosa gástrica. No se ha demostrado evidencia de que la asociación de fármacos no gastrolesivos o pluripatología provoque un incremento en el riesgo de sangrado digestivo $(3,7)$. No obstante Garrido y cols. realizan un estudio en el que se objetiva un incremento en el ingreso en las unidades de sangrantes de los pacientes con patología de base, aunque sigue siendo menor que las relacionadas con ulcus gastroduodenal e hipertensión portal (30).
La alta prevalencia de la dispepsia y del reflujo gastroesofágico que se presenta en los pacientes ingresados también contribuye a la administración de IBP (31-33) que, aun siendo una indicación de uso adecuada, en múltiples ocasiones no queda claramente recogido en la historia. Estos pacientes mantienen el tratamiento al alta sin presentar claras indicaciones. En el estudio realizado por Rey y cols. los síntomas por reflujo gastroesofágico demuestran tener un gran impacto sobre la utilización de recursos sanitarios y el absentismo laboral en España. Ello contrasta con la extendida opinión de que se trata de un trastorno de escasa importancia (32).

Actualmente el ingreso hospitalario no supone una mejoría en la adecuación del uso de los IBP frente a la utilización previa al ingreso $(\mathrm{p}=0,47)$, además, la probabilidad de que el IBP sea incluido en el tratamiento tras la estancia hospitalaria se multiplica por 2,36.

Los trabajos publicados sobre la implantación de guías de uso clínico en los servicios hospitalarios se han asociado a una mejor adecuación en la administración de estos fármacos, reduciendo el gasto farmacéutico que conlleva a la sobreutilización de los IBP y en la disminución de las interacciones y los efectos secundarios descritos con estos fármacos $(24,34)$.

En nuestro estudio queda reflejado, por tanto, el elevado porcentaje de pacientes que reciben tratamiento con IBP sin una indicación adecuada, sin que el ingreso sirva para su mejor administración o al menos sin que quede justificada al alta la toma del mismo (dispepsia, antiagregación en anciano con úlcera previa, etc.). Por ello, la opción más recomendable es la utilización de guías clínicas para el manejo correcto de los IBP, teniendo en cuenta las posibles modificaciones en las indicaciones que pueden resultar de las últimas publicaciones comentadas.

\section{BIBLIOGRAFÍA}

1. Flórez J. Farmacología de la secreción del aparato digestivo. En: Flórez J, Armijo JA, Mediavilla A, editores. Farmacología humana. $2^{a}$ ed. Barcelona: Masson; 1996. p. 684-5.

2. Robinson M, Horn J. Clinical pharmacology of proton pumps inhibitors. Drugs 2003; 63: 2739-54.

3. Nardino RJ, Vender RJ, Herbert PN. Overuse of acid-suppressive therapy in hospitalized patients. Am J Gastroenterol 2000; 95: 3118-22.

4. Ponce Romero M, Berenguer Lapuerta J. Indicaciones actuales de los inhibidores de la bomba de protones. Rev Clin Esp 2003; 203: 136-8.

5. Niklasson A, Bajor A, Bergendal L, Simren M, Strid H, Bjornsson E. Overuse of acid-suppressive therapy in hospitalized patients with pulmonary disease. Respir Med 2003; 97: 1143-50.

6. Naunton M, Peterson GM, Bleasel MD. Overuse of proton pump inhibitors. J Clin Pharm Ther 2000; 25: 333-40.

7. Noguerado Asensio A, Rodríguez Barrientos R, Zelaya Castro P, Sánchez Sempere A, Antuña Blanco F, Lutz García E, et al. Utilización de supresores de la secreción ácida en pacientes hospitalizados. An Med Interna 2002; 19: 557-60.

8. Conn HO, Poynard T. Corticosteroids and peptic ulcer: Meta-analysis of adverse events during steroid therapy. J Intern Med 1994; 236: 619-32.

9. Piper JM, Ray WA, Daugherty JR, et al. Corticosteroid use and peptic ulcer disease: Role of nonsteroidal anti-inflammatory drugs. Ann Intern Med 1991; 114: 735-40. 
10. Peura DA. Prevention of nonsteroidal anti-inflammatory drug-associated gastrointestinal symptoms and ulcer complications. Am J Med 2004; 117 (Supl. 5A): 63S-71S.

11. Rostom A, Dube C, Wells G, Tugwell P, Welch V, Jolicoeur E, et al. Prevention of NSAID-induced gastroduodenal ulcers. Cochrane Database Syst Rev 2002; (4): CD002296.

12. Mellemkjaer L, Blot WJ, Sorensen HT, Thomassen L, McLaughlin JK, Nielsen GL, et al. Upper gastrointestinal bleeding among users of NSAIDs: A population-based cohort study in Denmark. Br J Clin Pharmacol 2002; 53: 173-81.

13. Greenwald DA. Aging, the gastrointestinal tract, and risk of acid-related disease. Am J Med 2004; 117 (Supl. 5A): 8S-13S.

14. Hawkey CJ. Nonsteroidal anti-inflammatory drug gastropathy. Gastroenterol 2000; 119: 521-35.

15. Goldstein JL. Who needs prophylaxis of nonsteroideal anti-inflammatory drug-induced ulcers and what is optimal prophylaxis? Eur J Gastroenterol Hepatol 2000; 12 (Supl. 1): S11-5.

16. Pilotto A, Franceschi M, Leandro G, Paris F, Cascavilla L, Longo MG, et al. Proton-pump inhibitors reduce the risk of uncomplicated peptic ulcer in elderly either acute or chronic users of aspirin/non-steroidal anti-inflammatory drugs. Aliment Pharmacol Ther 2004; 20: 1091-7.

17. Johnsen SP, Sorensen HT, Mellemkjoer L, Blot WJ, Nielsen GL, McLaughlin JK, et al. Hospitalisation for upper gastrointestinal bleeding associated with use of oral anticoagulants. Thromb Haemost 2001; 86: 563-8.

18. Chryssostalis A, Marck G, Sibilia J, Chaussade S. Prevention of gastroduodenal complications in patients taking low-dose aspirin. Gastroenterol Clin Biol 2004; 28 (Spec. 3): C84-9.

19. Kimmey MB. Cardioprotective effects and gastrointestinal risks of aspirin: Maintaining the delicate balance. Am J Med 2004; 117 (Supl. $5 \mathrm{~A}): 72 \mathrm{~S}-8 \mathrm{~S}$

20. Nielsen GL, Sorensen HT, Mellemkjoer L, Blot WJ, McLaughlin JK, Tage-Jensen U, et al. Risk of hospitalization resulting from upper gastrointestinal bleeding among patients taking corticosteroids: A register-based cohort study. Am J Med 2001; 111: 541-5.

21. Mutlu GM, Mutlu EA, Factor P. Prevention and treatment of gastrointestinal complications in patients on mechanical ventilation. Am J Respir Med 2003; 2: 395-411.
22. Jung R, MacLaren R. Proton-pump inhibitors for stress ulcer prophylaxis in critically ill patients. Ann Pharmacother 2002; 36: 1929-37.

23. DePriest JL. Stress ulcer prophylaxis. Crit Care Med 2004; 32: 858-73.

24. Tryba M, Cook D. Current guidelines on stress ulcer prophylaxis. Drugs 1997; 54: 581-96.

25. Daley RJ, Rebuck JA, Welage LS, Rogers FB. Prevention of stress ulceration: Current trends in critical care. Crit Care Med 2004; 32: 2008-13.

26. Barletta JF, Erstad BL, Fortune JB. Stress ulcer prophylaxis in trauma patients. Crit Care 2002; 6: 526-30.

27. Faisy C, Guerot E, Diehl JL, Iftimovici E, Fagon JY. Clinically significant gastrointestinal bleeding in critically ill patients with and without stress-ulcer prophylaxis. Intensive Care Med 2003; 29: 1306-13.

28. Kantorova I, Svoboda P, Scheer P, Doubek J, Rehorkova D, Bosakova $\mathrm{H}$, et al. Stress ulcer prophylaxis in critically ill patients: A randomized controlled trial. Hepatogastroenterology 2004; 51: 757-61.

29. Allen ME, Kopp BJ, Erstad BL. Stress ulcer prophylaxis in the postoperative period. Am J Health Syst Pharm 2004; 61: 588-96.

30. Garrido A, Márquez JL, Guerrero FJ, Leo E, Pizarro MA. Cambios en la etiología, resultados y características de los pacientes con hemorragia digestiva aguda grave a lo largo del periodo 1999-2005. Rev Esp Enferm Dig 2007; 99: 275-9.

31. Castro Fernández M, García Díaz E, Larraona JL, et al. Eficacia de lansoprazol a dosis bajas en el tratamiento de la enfermedad por reflujo gastroesofágico no erosiva: influencia de la infección por Helicobacter pylori. Rev Esp Enferm Dig 2006; 98: 170-9.

32. Rey E, Moreno Elola-Olaso C, Rodríguez Artalejo F, et al. Impacto de los síntomas de reflujo gastroesofágico sobre el uso de servicios sanitarios y el absentismo laboral en España. Rev Esp Enferm Dig 2006; 98: 518-26.

33. Garza González E, Giasi González E, Martínez Vázquez MA, et al. Erradicación de H. pylori y su relación con la resistencia a los antibióticos y el estatus de CYP2C19. Rev Esp Enferm Dig 2007; 99: 71-5.

34. Mostafa G, Sing RF, Matthews BD, Pratt BL, Norton HJ, Heniford BT. The economic benefit of practice guidelines for stress ulcer prophylaxis. Am Surg 2002; 68: 146-50. 